\title{
Evaluating State Universities Websites Visibility in One Philippine Region Using Search Engine Optimization Tools
}

\author{
Marco Jr. N. Del Rosario \\ College of Computer Studies, Laguna State Polytechnic University, Brgy. Del Remedio, San Pablo City 4000, Laguna, Philippines
}

Received June 3, 2021; Revised July 9, 2021; Accepted August 22, 2021

\section{Cite This Paper in the following Citation Styles}

(a): [1] Marco Jr. N. Del Rosario , "Evaluating State Universities Websites Visibility in One Philippine Region Using Search Engine Optimization Tools," Computer Science and Information Technology, Vol. 9, No. 2, pp. 25 - 32, 2021. DOI: 10.13189/csit.2021.090201.

(b): Marco Jr. N. Del Rosario (2021). Evaluating State Universities Websites Visibility in One Philippine Region Using Search Engine Optimization Tools. Computer Science and Information Technology, 9(2), 25 - 32 . DOI: 10.13189/csit.2021.090201.

Copyright $\odot 2021$ by authors, all rights reserved. Authors agree that this article remains permanently open access under the terms of the Creative Commons Attribution License 4.0 International License

\begin{abstract}
This study analyses the visibility of the State Universities' website in Region 4A, Philippines during the pandemic wherein universities halted most of the physically performed transactions and started the delivery of services online through their websites. An attempt has been taken to help web content creators and developers to make their websites visible and easier to find in search engines. The websites' visibility was measured by collecting data through Search Engine Optimization tools, specifically Alexa, CheckPageRank, SEO Analyzer, and Moz Link Explorer. The collected data are the websites' global and national traffic rank, domain authority, PageRank, loading time and speed, daily pageviews, bounce rate, daily time on site, and site linking in. Websites were ranked based on how good or bad their SEO tool results. The analyzed result suggests that UPLB performed the most optimization since it ranks first in most of the tools. It was followed by BatSU and CvSU websites which consistently placed second and third in the rank list, respectively. The LSPU website occupies fourth place in the rank list, the SLSU website in fifth place, and the URS website in the last place. This study suggests that the least performing websites need to establish more high-quality website links and to create more user-needed contents to avoid an increase in bounce rate.
\end{abstract}

Keywords Search Engine Optimization, Management Information System, Web Service and Web Data Management, Website Visibility, State Universities

\section{Introduction}

The contagious coronavirus disease (COVID-19) caused a worldwide health crisis in early 2020, affecting not only the Philippines but the entire world. The Philippines' Department of Health (DOH) announced that on the $30^{\text {th }}$ of January 2020 the country recorded its first incident. However, the first local spread of the disease was confirmed on March 7 of the same year [1]. Consequently, the Philippine Government implemented a national Enhance Community Quarantine (ECQ) that requires Filipinos to stay at their home. Unfortunately, this also resulted in halting most of the country's operations, including the education sector. It was advised by the Department of Education (DepEd) and the Commission on Higher Education (CHED) that face-to-face classes on all levels shall be suspended [2,3]. However, despite the Enhance Community Quarantine implementation, the number of cases reported in the country is continuously adding up. As of August 7, 2020, the country has recorded 122,754 cases [4]. In response to the increasing number of cases, the government formulates and implements several types of Community Quarantine (Modified ECQ, General CQ, Modified CQ). However, regardless of the type, those who belong to the 
age group of 20 years old and below, above 60 years old, pregnant women, or those with comorbidities and health risks are not allowed to go out of the safety of their home $[5,6]$. This means that the implementation of the traditional enrollment process and teaching (face-to-face) is not permitted. To compensate, educational institutions pushed boundaries and explored new paths by using online technologies in implementing their enrollment and teaching activities $[2,5,7]$.

As an educational institution, having a website is an advantage. A website allows an establishment to convert its visitors into customers [8]. For a university, this will enable them to make their website visitor becomes its potential primary stakeholder, which is students. Moreover, it helps the students by providing several services such as, but not limited to, information dissemination, library services, and providing online learning delivery platform [9]. Websites can be easily accessed and located using their web address (URL). However, most potential visitors prefer to click a link rather than typing the web address in their browsers [10]. In this situation is where search engines come in since its main role is information seeking and retrieval to provide relevant information in their Search Engine Results Page (SERP) depending on the visitor's query [11]. Since many potential visitors rely on a search engine for their information needs, the visibility of a website in the SERP is very vital $[9,11,12]$. Increasing the visibility of a website in the SERP is important since most visitors are reluctant of reading past the first page of the SERP $[9,12]$. Thus, exploring search engines' potential is very important since search engines perform a critical part in directing users to the appropriate website. Studies have shown that search engines handled more than $90 \%$ of internet traffic $[9,11,13]$.

Search Engine Optimization (SEO) is among the several ways in how the visibility of a website in SERPs can be increased [10]. SEO refers to the process involving the activities undertaken to secure its visibility in the top spot on SERPs [11]. Making use of SEO to improve the performance and quality of a website is fundamental to getting a website to rank high in different search engines to attract possible customers $[10,12,14]$. It is the science of attracting web traffic to websites. Web traffic is said to be the life of any web-based business which measures its effectiveness at attracting visitors [9,15]. Users or potential customers spent most of their time on a website they enjoy; they read detailed texts or look at pictures. If a web content creator or developer wants to keep the visitors in, then the website's context and the loading time of the website become essential attributes [13].

Despite the fact that the vast majority of Philippine
State Universities and Colleges (SUCs) have websites, their visibility and searchability on search engine result pages are limited. In August 2020, using the generally used search engines which are Google, Yahoo, MSN, and Bing search engine, among the six state universities' websites in Region 4A (CALABARZON), none of it showed up on the search engine result page when the keywords "Universities in region $4 \mathrm{~A}$ " and "universities in CALABARZON" were searched. This situation and the ongoing effects of the global crisis served as the motivation for this research study.

The purpose of this study is to explore how search engine optimization (SEO) can be used as a strategy for increasing the visibility of state universities' websites on the internet. Specifically, this aims to measure the visibility of the Philippine state universities' websites in Region 4A using several search engine optimization tools. It determines the visibility of the state universities' websites on the Search Engine Results Page during the COVID-19 Pandemic in the country. Moreover, this study looks at how a specific webpage feature, such as indexing and backlinks, may be improved to enhance website visibility through search engines. It also finds out the reasons for the wide disparity in ranking between the websites. The results were analyzed and used to determine not just how the SUCs' websites differ from each other but also to show the degree of optimization that was performed. The researcher believes that there was no empirical evidence that a similar study has been conducted specifically on state universities and colleges' websites in the region.

\section{Methodology}

Official websites of the different state universities situated in the Region 4A of the Philippines were selected and subjected for comparative analysis. Table 1 presents the state universities, their location, and their corresponding web addresses.

The data were collected in July 2020, which is the typical start of the admission/enrollment period in Philippine universities. The proponent decided to use free online search engine optimization tools considering the chance that the selected universities will repeat or conduct the same process to measure how they have optimized their website. The following SEO tools utilized to analyze the six selected university websites were selected based on some published work/related literature: Alexa Metrics $[9,11,16,17,18,19,20]$, PageRank Checker [12,17,21], Patel's SEO Analyzer [16], and Moz Link Explorer (former Moz-Open Site Explorer) [18,22,23]. 
Table 1. List of State Universities in Region 4A - CALABARZON

\begin{tabular}{|c|c|c|c|}
\hline Name of Institution & Abbreviation & Website Address & Location \\
\hline Batangas State University & BatSU & batstate-u.edu.ph & Batangas City, Batangas \\
\hline Cavite State University & CvSU & cvsu.edu.ph & Indang, Cavite \\
\hline Laguna State Polytechnic University & LSPU & lspu.edu.ph & Sta. Cruz, Laguna \\
\hline Southern Luzon State University & SLSU & slsu.edu.ph & Lucban, Quezon Province \\
\hline University of the Philippines - Los Baños & UPLB & uplb.edu.ph & Los Baños, Laguna \\
\hline University of Rizal System & URS & urs.edu.ph & Tanay, Rizal \\
\hline
\end{tabular}

Alexa Metrics (alexa.com/siteinfo) is a free service that provides global metrics used for business development and several marketing strategies such as market research, analysis, and benchmarking [9]. This service accumulates user data from their clients who have downloaded their toolbar [17]. In this study, Alexa was used to collecting the global and national traffic ranking of each website, the daily pageviews per visitor, bounce rate, the total number of websites linking in, and the average daily time on the website.

PageRank Checker (checkpagerank.net) shows the website's popularity and calculates the position of a website in the Google search engine which is commonly called the Google page rank [12,17]. Most of the aspects measure by this tool were also similar to the other tools (e.g. Alexa Global, USA, and Reach rank, linking site, and Domain and Page Authority). Therefore, the researcher opted to rank using the overall Google Page rank score and cPR (presumed to stand for "CheckPageRank") score only.

Moz Link Explorer (moz.com/link-explorer) measures the website domain authority which is used to indicate how well or bad a website might rank on SERPs. Domain Authority refers to a search engine ranking score, typically ranging from 1 to 100 . These scores can be used in comparing websites. A higher score indicates that the "ranking strength" of the website is better than other sites [24]. In this study, Moz Link Explorer was used to measure the Domain Authority and the number of linking domains in every state university website.

This study used Neil Patel's SEO Analyzer (neilpatel.com/seo-analyzer/) to perform SEO analysis to analyze the aspects of loading time and speed depending on the device used (desktop or mobile).

After submitting the URL of the websites in the SEO tools and gathering the results, a rank was given to every website depending on how good or bad it was optimized on every metric. Also, the rank is based on how often the website's rank has been repeated. Then, an individual rank of each SUCs website was given based on the selected SEO tools. The individual rank of a website refers to the position it occupies in the list based on its performance in a particular SEO tool. In case that two or more websites are tied in a certain position in the list an averaged rank was given. Then a cumulative total of the websites' ranks from each SEO tool were computed and then taken to provide an overall ranking of the six SUCs' websites.

\section{Results and Discussion}

After using the previously mentioned tools to evaluate all the websites, the retrieved results are presented in tables.

\subsection{Analysis using Alexa Metrics}

Table 2 shows the results of the Alexa Metrics for the selected state universities' websites. The majority of the websites had low global traffic rankings, as seen by their high-numbered ranks. The best-ranked state university website placed 326,069th globally, while the least-ranked website placed 3,732,666th globally. A global rank below 100,000 is considered to be a "great" position and a rank more than that is considered to be lacking in data $[19,25]$. This indicates that none of the websites were given a relatively great ranking, and all the selected universities' websites were ranked poorly. Table 2 also shows that three of the universities have missing values. This happens because Alexa Ranking does not receive enough data for the past 90 days ( 3 months) from their sources to make a ranking [20]. This can be attributed to why three of those websites did not place higher in the global ranking compared to the three websites with national rank. A national rank of lower than 5,000 is projected as having a good performance in that attribute [20]. It can be seen in Table 2 that only two websites have a rank lower than 5,000. The researcher also considered looking at the consistency of the websites' global rank and national rank. However, the consistency between the national rank and global rank of all six state universities and colleges' websites cannot be observed since there are missing values. Nevertheless, if the universities' websites without the national ranking were omitted, consistency was observed on the remaining three universities' websites. But still, the researcher recommends that every website should apply in Alexa to be certified in order to be directly measured by its site metrics.

The visibility of a website in SERP can be increased by the number of web pages indexed in a search engine. Thus, 
an increase in indexed web pages has a significant relationship with the number of daily pageviews a website receives. Therefore, it is recommended that universities' websites should index more web pages on search engines. As presented in Table 2, 1spu.edu.ph (LSPU) ranked first in the metric by having an average pageview of 5 . It was followed by urs.edu.ph (URS), batstate-u.edu.ph (BatSU), cvsu.edu.ph (CvSU), uplb.edu.ph (UPLB), and then slsu.edu.ph (SLSU) with an average pageview of 4, 3, 2.8, 2.2, and 1, respectively. According to a study by Mandal and Das [26], there is a strong positive relationship between the average daily pageviews and the average time on site. It can be interpreted that since lspu.edu.ph had a high average pageviews, it will also have a high time on site. On the contrary, since slsu.edu.ph had a low average pageview, it will also have a low average time on site.

Time spent on site is the metric that reveals whether a website is serving its purpose and engaging its visitors [27]. In terms of the average daily time on site, five of the universities' websites have data. The website with the longest daily time on site was batstate-u.edu.ph (BatSU) with 7:54 (m:s), while the shortest daily time on site was 2:44 (m:s) of uplb.edu.ph (UPLB). Since site visitors were staying in an acceptable amount of time on the website (more than 2 minutes [27,28]), it is reasonable to assume that all websites were performing well in this metric. It can be interpreted that there is no evident usability problem in the organization of website content or its information architecture.

Stanis [27] suggests that the daily time on site can also be attributed to a website bounce rate. Bounce rate is a metric used to measure the user's satisfaction in finding the exact content on a website. Fundamentally, it refers to the proportion of website visitors that leave the website once the home page was loaded and without even navigating to any other webpage on the site [28]. A higher percentage in bounce rate can be interpreted as lower user satisfaction. Similar to the national rank, three of the universities' websites do not have a score. As indicated in Table 2, batstate-u.edu.ph (BatSU) had the lowest bounce rate value among the three sites of $36.4 \%$ which is considered an excellent percentage based on Stanis' [27] study results. On the other hand, cvsu.edu.ph (CvSU) had a bounce rate value of $45.5 \%$ and uplb.edu.ph (ULB) had the highest bounce rate value of $55.9 \%$, which are both considered to be in an average bounce rate. Such bounce rates were attained when a visitor is uninterested in the content or struggling in using the website. Moreover, Hasan, Morris, and Probets [28] emphasized that having a high bounce rate and low pageviews can be a result of potential website navigational problems.

The number of sites that are linked to the being analyzed website is referred to as the total site linking in [18]. As shown in Table 2, uplb.edu.ph (UPLB) has the most sites linking in (471). In contrast, slsu.edu.ph (SLSU) had the lowest number of sites linking in (18). It is assumed that a website with the highest number of sites linking in has a higher probability to be included in SERPs compared to the website with a lower number of sites linking in.

Table 2. Alexa Metrics of State Universities Websites

\begin{tabular}{|c|c|c|c|c|c|c|}
\hline Website & Global Rank & PH Rank & $\begin{array}{c}\text { Daily Pageviews } \\
\text { per Visitor }\end{array}$ & $\begin{array}{c}\text { Daily Time on } \\
\text { Site (m:s) } \\
\end{array}$ & $\begin{array}{c}\text { Bounce } \\
\text { Rate }\end{array}$ & $\begin{array}{c}\text { Total site } \\
\text { Linking in }\end{array}$ \\
\hline batstate-u.edu.ph & 378460 & 2867 & 3.0 & $7: 54$ & $36.4 \%$ & 117 \\
\hline cvsu.edu.ph & 1128706 & 9548 & 2.8 & $5: 28$ & $45.5 \%$ & 105 \\
\hline lspu.edu.ph & 1589058 & - & 5 & $6: 41$ & - & 19 \\
\hline slsu.edu.ph & 3732666 & - & 1 & - & - & 18 \\
\hline uplb.edu.ph & 326069 & 1630 & 2.2 & $2: 44$ & $55.9 \%$ & 471 \\
\hline urs.edu.ph & 2374347 & - & 4 & $6: 55$ & - & 44 \\
\hline
\end{tabular}

Table 3. Rank List based on Alexa Metrics Result

\begin{tabular}{|c|c|c|c|c|c|c|c|}
\hline Website & $\begin{array}{c}\text { Global } \\
\text { Rank }\end{array}$ & PH Rank & $\begin{array}{c}\text { Daily Pageviews } \\
\text { per Visitor }\end{array}$ & $\begin{array}{c}\text { Daily Time on } \\
\text { Site (m:s) }\end{array}$ & $\begin{array}{c}\text { Bounce } \\
\text { Rate }\end{array}$ & $\begin{array}{c}\text { Total site } \\
\text { Linking in }\end{array}$ & Rank \\
\hline batstate-u.edu.ph & 2 & 2 & 3 & 1 & 1 & 2 & 2 \\
\hline cvsu.edu.ph & 3 & 3 & 4 & 4 & 2 & 3 & 3 \\
\hline lspu.edu.ph & 4 & 5 & 1 & 3 & 5 & 5 & 4 \\
\hline slsu.edu.ph & 6 & 5 & 6 & 6 & 5 & 6 & 6 \\
\hline uplb.edu.ph & 1 & 1 & 5 & 5 & 3 & 1 & 1 \\
\hline urs.edu.ph & 5 & 5 & 2 & 2 & 5 & 4 & 5 \\
\hline
\end{tabular}


Based on the websites' most frequently repeated ranks on the six criteria, the final ranking using the Alexa Metrics is as presented in Table 3. In general, uplb.edu.ph (UPLB) ranked first in the Alexa Metrics. It topped all the other universities' websites in terms of global rank, national rank, and total site linking in. BatSU came in second with two rank one places, based on bounce rate and daily time on site criteria. Despite not ranking first in any of the criteria, cvsu.edu.ph (CvSU) ranked third because of its consistency. lspu.edu.ph (LSPU) had its lead compared to urs.edu.ph (URS) because of its rank 1 position in the number of average daily page views criteria. Unfortunately, slsu.edu.ph (SLSU) was placed at the last of the rank due to the incomplete value found in the Alexa Metrics results.

\subsection{Analysis using PageRank Checker}

Another SEO tool used in this study is the PageRank Checker. The page rank of the universities' websites has been retrieved and presented in Table 4. Google PageRank is represented in 1 to 10 values [21, 29]. As shown in Table 4, uplb.edu.ph (UPLB) has the highest result with a value of 5, thus placing it in the first spot of the rank. On the other hand, the remaining five websites have a similar Google PageRank value of 3, thus making it difficult to determine their ranking. Fortunately, PageRank Checker provides another metric in a form of cPR (assumed to be an abbreviation of CheckPageRank). cPR score ranges from 0.0 to 10.0. It weights other ranking metrics along with the previous google ranking algorithm. Regarding the result, the consistency between the Google PageRank score and cPR score was observed. As shown in the table, uplb.edu.ph still has the highest score in the metric (5.2), followed by batstate-u.edu.ph (3.7), cvsu.edu.ph (3.6), lspu.edu.ph (3.2), slsu.edu.ph (3.1), and then urs.edu.ph (3.0).

Table 4. PageRank Checker Result

\begin{tabular}{|c|c|c|c|}
\hline Website & $\begin{array}{c}\text { Google } \\
\text { PageRank }\end{array}$ & cPR Score & Rank \\
\hline batstate-u.edu.ph & 3 & 3.7 & 2 \\
\hline cvsu.edu.ph & 3 & 3.6 & 3 \\
\hline 1spu.edu.ph & 3 & 3.2 & 4 \\
\hline slsu.edu.ph & 3 & 3.1 & 5 \\
\hline uplb.edu.ph & 5 & 5.2 & 1 \\
\hline urs.edu.ph & 3 & 3.0 & 6 \\
\hline
\end{tabular}

\subsection{Analysis using Moz Link Explorer}

Domain Authority is a predictive measure of how well a website, both domain and its subdomains, will rank and be visible on the Search Engine Result Pages [21,22]. Ruhela, Kumar, and Kumar [23] added that it is represented by a score ranging from 1 to 100 , with a higher score indicating a higher possibility of showing at the top of the SERPs.

As shown in Table 5, it was found out that based on the domain authority of the universities' website, UPLB is in the highest position with a score of $48 / 100$, followed by BatSU with 31/100 closely followed by CvSU and URS with a score 28/100 and 27/100, respectively. It is followed by LSPU with a score of $25 / 100$, while SLSU at the lowest position has a score of 22/100. Onaifo and Rasmussen [11] suggest that a higher Domain Authority score can be achieved by increasing the number of high-quality linking domains.

Table 5. Moz Link Explorer Result

\begin{tabular}{|c|c|c|c|}
\hline Website & $\begin{array}{c}\text { Domain } \\
\text { Authority }\end{array}$ & $\begin{array}{c}\text { Linking } \\
\text { Domains }\end{array}$ & Rank \\
\hline batstate-u.edu.ph & $31 / 100$ & 581 & 2 \\
\hline cvsu.edu.ph & $28 / 100$ & 469 & 3 \\
\hline 1spu.edu.ph & $25 / 100$ & 231 & 5 \\
\hline slsu.edu.ph & $22 / 100$ & 279 & 6 \\
\hline uplb.edu.ph & $48 / 100$ & $5 \mathrm{k}$ & 1 \\
\hline urs.edu.ph & $27 / 100$ & 260 & 4 \\
\hline
\end{tabular}

Table 5 indicates that the UPLB website was linked to more than 5000 domains, which ranked the university in the first place. Moreover, it can also be interpreted that linking domains relatively affect the score of the domain authority. The consistency between the domain authority score and the number of linking domains can also be observed with BatSU (581), CVSU (469), and URS (260). However, in the case of the lowest ranking universities websites, LSPU has a higher domain authority score compared to SLSU but a lower number of linking domains. This could happen because of other factors not shown in the table. Linking Domains is the number of high-quality websites that are linked to a website. These high-quality websites that are linked to a website or web pages will also send relevant traffic. According to Yalçın and Köse [30], search engines like Google considers every link present in a website as a positive step for the related website. However, some search engines check the contents of the linked web pages. Thus, Domain Linking or Link building which is a technique that has a significant influence on the ranking of websites for most search engines should be considered for optimization bearing in mind that the webpages must have similar or related content.

\subsection{Analysis using Patel's SEO Analyzer}

Patel's SEO Analyzer has been used to analyze and compare universities' websites based on load speed. Since everyone tends to access the internet and search using their smartphones, search engines addressed this by releasing a "mobile-first" algorithm update [29]. This update considers a mobile-friendly website to provide 
merits to that website to rank higher in SERPs. For that reason, SEO tools provide metrics in measuring the speed of loading a mobile-friendly website. Load Speed (measured in time) refers to the average required time to load a website and its components to a browser [12].

As shown in Table 6, UPLB and LSPU have the shortest loading time of 6 seconds, followed by $\mathrm{CvSU}$ and SLSU with 10 seconds loading time, closely followed by URS with 11 seconds. However, BatSU has the longest loading time of 51 seconds. The researcher also considered looking at the consistency of the websites' load speed on Desktop and Mobile.

Table 6. Patel's SEO Analyzer Result

\begin{tabular}{|c|c|c|c|}
\hline Website & $\begin{array}{c}\text { Load Speed } \\
\text { (Desktop) }\end{array}$ & $\begin{array}{c}\text { Load Speed } \\
\text { (Mobile) }\end{array}$ & Rank \\
\hline batstate-u.edu.ph & $18 \mathrm{~s}$ & $51 \mathrm{~s}$ & 6 \\
\hline cvsu.edu.ph & $3 \mathrm{~s}$ & $10 \mathrm{~s}$ & 3.5 \\
\hline 1spu.edu.ph & $2 \mathrm{~s}$ & $6 \mathrm{~s}$ & 1 \\
\hline slsu.edu.ph & $3 \mathrm{~s}$ & $10 \mathrm{~s}$ & 3.5 \\
\hline uplb.edu.ph & $3 \mathrm{~s}$ & $6 \mathrm{~s}$ & 2 \\
\hline urs.edu.ph & $12 \mathrm{~s}$ & $11 \mathrm{~s}$ & 5 \\
\hline
\end{tabular}

It can be observed that the four universities' websites CVSU, LSPU, SLSU, and UPLB - were consistent in having a fast-loading speed ranging from 2-3 seconds on desktop and 6-10 seconds on mobile. Consistency was also observed in BatSU for having the slowest loading speed in both desktop and mobile criteria with 18 seconds and 51 seconds, respectively. URS did not show a large gap in loading speed in both criteria. Table 6 also shows what the rank would look like if the SUCs' websites are given ranks based on load speed criteria in Patel's SEO Analyzer results.

\subsection{Overall Rank}

After subjecting to the tools and getting the results, the total rank of all universities' websites was calculated and ranked. As shown in Table 7, the website of UPLB ranked first on three SEO tools in this ranking, followed by the
BatSU website which placed second on three SEO tools. Similar to BatSU, the website of CvSU ranked third on three SEO tools. The LSPU website ranked fourth, followed by the URS website, and lastly the SLSU website which ranked sixth on three SEO tools.

\section{Conclusion}

Since none of the six state universities' websites in the Philippines' Region 4A was visible on the SERPs during the COVID-19 Pandemic, exploring the benefits of conducting optimization techniques on those websites is greatly needed. Search Engine Optimization (SEO) tools such as Alexa, PageRank Checker, SEO Analyzer, and Moz Link Explorer were applied to comparatively evaluate the websites of the State Universities in Region 4A, Philippines. Using each SEO tool, selected websites were individually ranked in an attempt to aid SUCs web developers and content creators to make their websites more visible and easier to find in search engines.

The results showed that the UPLB website has the highest degree of optimization done compared to other websites since it aced in most of the tools and its corresponding metrics. It was followed by the BatSU website, which consistently performed well in most tools except in loading speed in both desktop and mobile devices where it placed last. CvSU website, which consistently placed third in all tools, needs to consider optimizing their loading time, increase the time spent of users on the website, and consider indexing more webpages on search engines to be at par or exceed the UPLB website results. On the other hand, LSPU, SLSU, and URS websites do not have enough data, as shown in Alexa metrics results, which indicates that they need to perform the most work in optimizing their website. The study suggests that the websites lack in building high-quality website links, and it needs to create more user-needed contents to avoid an increase in bounce rate. This study provides recommendations for the state universities in region 4A in the Philippines on how they can further improve the visibility of their websites.

Table 7. Overall Rank

\begin{tabular}{|c|c|c|c|c|c|}
\hline Website & Alexa & PageRank Checker & SEO Analyzer & $\begin{array}{c}\text { Moz Link } \\
\text { Explorer }\end{array}$ & Overall Rank \\
\hline batstate-u.edu.ph & 2 & 2 & 6 & 2 & 2 \\
\hline cvsu.edu.ph & 3 & 3 & 3.5 & 3 & 3 \\
\hline lspu.edu.ph & 4 & 4 & 1 & 5 & 4 \\
\hline slsu.edu.ph & 6 & 5 & 3.5 & 6 & 6 \\
\hline uplb.edu.ph & 1 & 1 & 2 & 4 & 1 \\
\hline urs.edu. ph & 5 & 6 & 5 & & 5 \\
\hline
\end{tabular}




\section{Future Research}

This study provides opportunities for more research on SEO and web visibility of universities' websites. In the future, the author plans to implement the method applied to a wider scope expecting that the research findings will greatly help and enable web content creators and developers to enhance the visibility and ranking of their website in the search engine result pages. Furthermore, the methodology can be applied to specific services given by the universities like library and student support services to further increase effective optimization of the websites. Also, the author plans to explore other factors to be considered in designing websites such as website usability and content quality. Overall, this study has improved the understanding of the impact of search engines and search engine optimization techniques in increasing the website's exposure or visibility to its visitors.

\section{Acknowledgements}

The author appreciates the assistance provided by the College of Computer Studies at Laguna State Polytechnic University in conducting this study.

\section{REFERENCES}

[1] "Coronavirus disease (COVID-19) in the Philippines". World Health Organization, https://www.who.int/philippin es/emergencies/covid-19-in-the-philippines (accessed Aug. $12,2020)$

[2] Briones, L., "Guidelines for the Remainder of School Year 2019-2020 In Light of COVID-19 Measures", Department of Education, https://www.deped.gov.ph/wp-content/uploa ds/2020/03/DM_s2020_042-20200315_Guidelines-for-Re mainder-of-SY-2019-2020-COVID-19.pdf (accessed Jul. 5, 2021)

[3] De Vera III, J., "CHED COVID Advisory No. 2 Guidelines for Prevention, Control, and Mitigation of the Spread of Coronavirus Disease 2019 (COVID-19) in Higher Education Institutions (HEIs)", Commission on Higher Education, https://ched.gov.ph/wp-content/uploads/ CHED-COVID-2019-Advisory-No.-2.pdf (accessed Jul. 28, 2020)

[4] "DOH COVID-19 Bulletin \# 146", Department of Health, from https://www.doh.gov.ph/node/23948 (accessed Aug. 8, 2020)

[5] De Vera III, J., "CHED COVID Advisory No. 7 Guidelines for Prevention, Control, and Mitigation of the Spread of Coronavirus Disease 2019 (COVID-19) in Higher Education Institutions (HEIs)", Commission on Higher Education, https://ched.gov.ph/wp-content/uploads/ CHED-ADVISORY-7-final.pdf (accessed Jul. 28, 2020)

[6] "Omnibus Guidelines on the Implementation of Community Quarantine in the Philippines with
Amendments as of July 02, 2020”, Inter-Agency Task Force for the Management of Emerging Infectious Diseases, https://doh.gov.ph/sites/default/files/health-update/omnibu s-guidelines-on-the-implementation-of-community-quarant ine-in-the-philippines0702.pdf (accessed Jul. 5, 2021)

[7] Llego, M., "DepEd Enrollment Manual for Philippine Basic Education Schools for School Year 2020-2021", Teacher PH,https://www.teacherph.com/deped-enrollment-manualphilippine-basic-education-schools/ (accessed Aug. 8, 2020)

[8] Diffily, S., "Website Development", The website manager's handbook. Lulu.com, 2006.

[9] Weideman, M., "Comparative analysis of homepage Website visibility and academic rankings for UK universities," Information Research: An International Electronic Journal, vol. 18, no. 4, pp. n4, 2013. http://digitalknowledge.cput.ac.za/bitstream/11189/2709/3/ Weideman_Melius_FID_2013.pdf

[10] Arsenault, T., Feeny, D., "Your website: greater visibility and return of investment through search engine marketing," DESIDOC Journal of Library \& Information Technology, vol. 28, no. 4, pp. 79, 2008. https://publications.drdo.gov.i n/ojs/index.php/djlit/article/view/200/108

[11] Onaifo, D., \& Rasmussen, D., "Increasing libraries' content findability on the web with search engine optimization", Library Hi Tech, vol. 31 no. 1, pp. 87-108, 2013. DOI: $10.1108 / 07378831311303958$

[12] Al-Ananbeh, A. A., Ata, B. A., Al-Kabi, M., Alsmadi, I., "Website usability evaluation and search engine optimization for eighty Arab university websites". Basic Science \& Engineering, vol. 21, no. 1, pp. 107-122, 2012. http://86.108.11.14/bitstream/123456789/2054/1/595162.p df

[13] Egri, G., Bayrak, C., "The role of search engine optimization on keeping the user on the site", Procedia Computer Science, vol. 36, pp. 335-342, 2014. DOI: 10.1016/j.procs.2014.09.102

[14] Kent, P., "Beating the Competition - Planning a Powerful Search Engine Strategy", Search engine optimization for dummies, $5^{\text {th }}$ ed, John Wiley \& Sons, 2012, pp. 49-65.

[15] Davis, H., "Search engine optimization - SEO's Evolution", Search Engine Optimization, O'Reilly Media, Inc., 2006, pp $1-3$.

[16] Vyas, C., "Evaluating state tourism websites using Search Engine Optimization tools", Tourism Management, vol. 73, pp. 64-70, 2019. DOI: 10.1016/j.tourman.2019.01.019

[17] Dadkhah, M., Bianciardi, G., "Fake Universities as an Emerging Issue". International Journal of Web-Based Learning and Teaching Technologies, vol. 11, no. 4, pp. 49-52, 2016. DOI: 10.4018/IJWLTT.2016100104

[18] Jayasekara, P. K., "Webometric Analysis on Leading Course Providers in MOOC: A Study Based on Alexa Ranking", 11th International CALIBER-2017, pp. 342-350, 2017. https://ir.inflibnet.ac.in/bitstream/1944/2101/1/36.pd f

[19] Hanief Bhat, M., "Evaluating Indian newspaper web sites using Alexa Internet", Library Review, vol. 62, no. 6/7, 
pp.398-406, 2013. https://doi.org/10.1108/LR-01-2013-00 10

[20] Muthuraja, S., Veerabasavaiah, M.," An Evaluation of Kannada News Paper Websites Using Alexa Internet Tool: A Webometric Study", International Journal of Library and Information Studies, vol. 8, no. 1, pp. 202-09, 2019. https://www.ijlis.org/articles/an-evaluation-of-kannada-ne ws-paper-websites-using-alexa-internet-tool-a-webometric -study.pdf

[21] Hossen, M. K., Ayman, A., "JRanker: An Approach to Evaluate the Prestige of a Journal Using PageRank and Alexa Rank along with Impact Factor", 1st International Conference on Information and Communication Technology (ICAICT), 2019.

[22] Aker, A., Vincentius, K., Bontcheva, K., "Credibility and Transparency of News Sources: Data Collection and Feature Analysis", Proceedings of the Third International Workshop on Recent Trends in News Information Retrieval (NewsIR@ SIGIR2019),pp. 15-20, 2019.http://ceur-ws.or g/Vol-2411/paper3.pdf

[23] Verma, M. K., Brahma, K., "A webometric analysis of National Libraries' websites in South Asia", Annals of Library and Information Studies (ALIS), vol. 64, no. 2, pp. 116-124, 2017.http://14.139.47.23/index.php/ALIS/article/ view/15610

[24] Ruhela, A., Kumar, M., Kumar, S., "Webometric study of the Fisheries Institutions Websites of Indian council of
Agricultural Research", International Journal of Library and Information Studies, vol. 7, no. 4, pp. 115-123, 2017. https://www.ijlis.org/articles/webometric-study-of-the-fish eries-institutions-websites-of-indian-council-of-agricultura 1-research.pdf

[25] "Domain Authority", Moz, Inc., https://moz.com/learn/seo/ domain-authority (accessed Aug. 31, 2020)

[26] Mandal, S., Das, A., "Correlation between Daily Page Views and View Duration: An Exploration of National Library Websites", SRELS Journal of Information Management, vol. 55, no. 5, pp. 247-253, 2018. DOI: $10.17821 /$ srels/2018/v55i5/132073

[27] Stanis, J., "How to Improve Website Bounce Rate and Time on Page", Weidert Group, https://www.weidert.com/blog/a verage-bounce-rate-and-time-on-page (accessed Aug. 31, 2020)

[28] Hasan, L., Morris, A., Probets, S., "Using Google Analytics to evaluate the usability of e-commerce sites", International Conference on Human Centered Design, vol 5619, pp. 697-706, 2009. DOI: 10.1007/978-3-642-02806-9_81

[29] Bitler, A., "Backlink Building in 2018: Check Page Rank!", Check Page Rank,https://checkpagerank.net/blog/seo/back link-building-in-2018.php (accessed Aug. 31, 2020)

[30] Yalçın, N., Köse, U., "What is search engine optimization: SEO?", Procedia-Social and Behavioral Sciences, vol. 9, pp. 487-493, 2010. DOI: doi.org/10.1016/j.sbspro.2010.12.185 